\title{
Indicated Cesarean Delivery
}

National Cancer Institute

\section{Source}

National Cancer Institute. Indicated Cesarean Delivery. NCI Thesaurus. Code C114142.

A cesarean delivery that is performed for specific, medical or obstetric indications, which may be scheduled or unscheduled. 